PAPER

\title{
Unilateral posterior parietal lobe lesions disrupt kinaesthetic representation of forearm orientation
}

\author{
W G Darling, R Bartelt, M Rizzo
}

J Neurol Neurosurg Psychiatry 2004;75:428-435. doi: 10.1136/jnnp.2003.026138

See end of article for authors' affiliations ....................

Correspondence to: Dr W G Darling, Department of Exercise Science, 526 Field House, University of lowa, lowa City, lowa 52242, USA; warren-darling@ viowa.edu

Received 19 August 2003 Accepted 19 August 2003
Objective: To apply the lesion method to assess neuroanatomical substrates for judgments of forearm orientation from proprioceptive cues in humans.

Methods: Participants were 15 subjects with chronic unilateral brain lesions and stable behavioural deficits, and 14 neurologically normal controls. Subjects aligned the forearm to earth fixed vertical and trunk fixed anterior-posterior (A-P) axes ("straight ahead"), with the head aligned to the trunk and with head and shoulder orientations varied on each trial.

Results: Most subjects with posterior parietal lobe lesions made larger variable errors than controls in aligning the forearm to the earth fixed vertical axis and the trunk A-P axes, whether the head was held upright or oriented in different positions. Lesion subjects and controls made similar constant errors for aligning the forearm to gravitational vertical. Variable error magnitude correlated positively with greater lesion volume of right and left superior parietal lobules (SPL), but not with lesions in other brain areas. Larger variable errors for aligning the forearm to the trunk fixed A-P axis were also correlated with the volume of SPL lesions, but constant error magnitude correlated with larger volume lesions in premotor areas, inferior parietal lobules, and posterior regions of the superior temporal gyri, but not with SPL lesion volume.

Conclusions: The findings suggest that the right and left superior and inferior parietal lobules, posterior superior temporal gyri, and premotor areas play a role in defining higher level coordinate systems for specifying orientation of the right and left forearm.
$\mathrm{P}$ rogramming accurate reaches to visual targets depends on awareness of upper limb segment orientation. Subjects who have no sensations related to upper limb segment orientations make highly variable reaches to visual targets. ${ }^{12}$ Studies of kinaesthetic perception have shown that neurologically normal humans align the forearm more accurately to the earth fixed vertical axis (fig 1A) and the trunk fixed anterior-posterior (A-P) axis (fig 1B) than to other head and trunk axes. ${ }^{3}$ These two axes are also used by the visual perceptual system to specify object orientations at the perceptual level. ${ }^{56}$ Use of the same axes by these two sensory systems simplifies transformation of visual information into an upper limb coordinate system, which allows linear estimates of arm and forearm orientations for target acquisition. ${ }^{78}$ Motor commands to guide the upper limb accurately to an orientation suitable for target acquisition are thought to depend on the difference between current upper limb orientation and an orientation required for target acquisition estimated from visual coordinates of the target. ${ }^{9}$ Such sensorimotor transformations probably occur within a frontoparietal network that includes the posterior parietal cortex. ${ }^{10}$ The posterior parietal lobules (PPL) may play a key role in these coordinate transformations for programming reaching movements because of their dense inputs from both visual cortex and somatosensory areas in the postcentral gyrus.

In this study we examined the effects of lesions in different regions of the PPL (inferior and superior parietal lobules) and areas of temporal and frontal lobes on perceptual representation of forearm orientation in humans. Because of its extensive visual, vestibular, and proprioceptive inputs it is likely that the PPL is involved in transforming sensations of joint angles, which represent a lower level coordinate system for specifying upper limb orientations, into perception of upper limb segment orientations relative to the kinaesthetic coordinate system axes. The posterior region of the superior temporal gyrus ${ }^{11}$ and the frontal premotor areas $(6,44)^{12}$ may also be involved because of their role in higher level spatial perception. We hypothesised that lesions of these areas would disrupt higher level kinaesthetic perception of the earth fixed vertical and the trunk fixed A-P axes, especially when head and shoulder orientations are varied. We used multiple linear regression analysis to assess whether lesions of these brain regions contribute to larger errors in higher level kinaesthetic perception.

\section{METHODS}

\section{Subjects}

Fourteen neurologically normal controls (six male, eight female; mean (SD) age 66 (5.9) years), 15 individuals with chronic stage lesions (at least three months post-lesion) involving the posterior parietal lobe (table l; age 56.6 (17.6) years), and eight individuals with cerebral lesions outside the parietal lobe (table 2; age 51 (16.1) years) participated in the experiments. The lesion locations specified in tables 1 and 2 were defined from magnetic resonance imaging (MRI) or computed tomography using standard techniques. All subjects with brain lesions had neuropsychological testing, including tests for hemineglect (tables 1 and 2). Only one subject, who had a right sided PPL lesion that extended into the mesial deep right superior parietal lobule (SPL) paraventricular area, showed contralateral hemineglect (table 1 , LPPL-9).

All subjects signed informed consent forms approved by the University of Iowa institutional review board.

Abbreviations: PPL, posterior parietal lobule; PSTG, posterior superior temporal gyrus; SPL, superior parietal lobule 
A

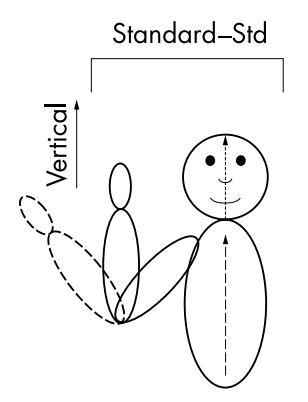

Perception of vertical

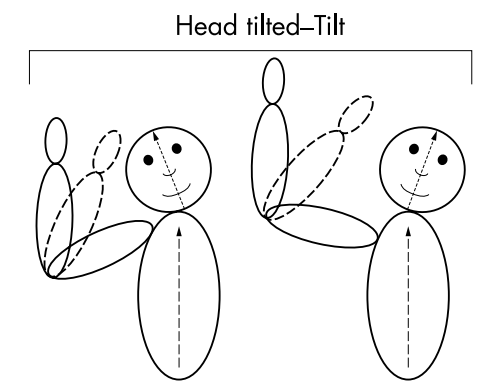

B

Perception of trunk-fixed A-P axis

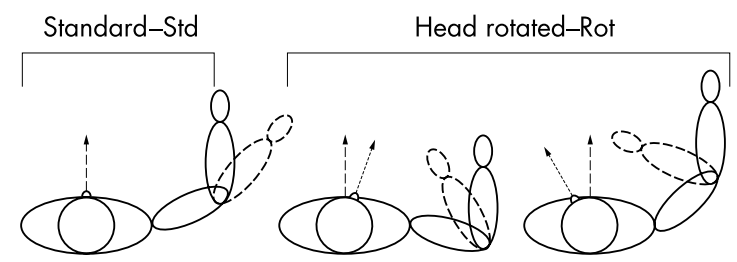

Figure 1 Experimental setup and tasks. The trunk (ellipses - dashed lines indicate longitudinal axis in panel $A$ and anterior-posterior (A-P) axis in panel B), head (circles - dotted lines indicate longitudinal axis in $A$ and $A-P$ axis in B), and upper limb segments (ellipses) are shown from an anterior view in $A$ (align forearm to vertical) and from a top view in $B$ (align forearm to trunk A-P axis). Possible head and arm (shoulder) orientations are shown for initial (dashed) and final (solid lines) upper limb orientations in the standard condition (left side) and head orientation varied condition (right side).

\section{Kinaesthetic perception of earth fixed vertical}

The blindfolded subject sat in a comfortable dental chair with the forearm supported on a table and performed elbow flexion movements to position the forearm parallel to the earth fixed vertical axis. The head and trunk were tilted backward about $0.3 \mathrm{rad}$ when seated in the chair. Vertical was described as perpendicular to the floor or parallel to the direction a weight would fall when released from a stationary position. The experimenter also demonstrated the task before the experiment began, to ensure that all subjects understood the instructions.

There were two experimental conditions:

(1) standard: five to eight trials of aligning the forearm to vertical; with the head in normal alignment relative to the trunk, the arm (humerus) was abducted approximately $90^{\circ}$;

(2) varied: 20 trials with varied head posture (head tilted to left or right by variable amounts on each trial) and varied shoulder orientation (by adjusting the height of the chair so that the subject's elbow angle at vertical changed on each trial to prevent them using a remembered elbow angle to align the forearm to vertical (fig 1)).

The initial elbow joint angle was varied on each trial in both conditions. When time permitted, both upper limbs were tested. However, if time constraints prevented testing both arms in a subject with a brain lesion, we tested the limb on the side ipsilateral to the lesion because we were most interested in global, or bilateral, effects of the lesions on high level kinaesthetic perception. Both upper limbs were tested in 11 of 14 controls, as well as in four of seven subjects with lesions outside the posterior parietal lobe and in 10 of 14 subjects with lesions of the posterior parietal lobe.

\section{Kinaesthetic perception of the trunk fixed A-P axis}

This task differed from the previously described task in that the subject's forearm rested on a horizontal manipulandum that allowed elbow flexion and extension about a nearly frictionless ball bearing pivot, and shoulder horizontal flexion and extension about a joint with friction to maintain a constant shoulder angle. The subject was instructed to align the forearm by elbow flexion or extension parallel to the trunk fixed A-P axis, which was described as an imaginary line extending straight forward from the trunk or sternum. Note that this task differs from previously described tasks

Table 1 Ages, lesion locations, and neglect ratings for individuals with posterior parietal cortex lesions

\begin{tabular}{|c|c|c|c|c|c|}
\hline \multirow[b]{2}{*}{ Subject group* } & \multirow[b]{2}{*}{ Age (years) } & \multirow[b]{2}{*}{ Sex } & \multirow[b]{2}{*}{ Locationt, sizeł and cause§ of lesion } & \multicolumn{2}{|c|}{ Neglect ratings $\Phi$} \\
\hline & & & & Acute & Chronic \\
\hline RPPL-1 & 83 & M & F06(2), F08(2), T08(3), T09(3), T12(2), P01(2), 101(3), 102(3); CVA & 1 & 1 \\
\hline RPPL-2 & 52 & M & 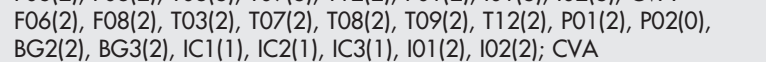 & $N T^{* *}$ & 1 \\
\hline RPPL-3 & 28 & $\mathrm{~F}$ & $\begin{array}{l}\mathrm{T} 04(2), \mathrm{TO} 6(2), \mathrm{T} 10(2), \mathrm{PO} 1(2), \mathrm{PO} 2(2), \mathrm{O} 05(2), \mathrm{TH} 1(2), \mathrm{TH} 2(2), \mathrm{TH} 3(2) \text {, } \\
\mathrm{TH} 4(2) ; \mathrm{CVA}\end{array}$ & 3 & 1 \\
\hline RPPL-4 & 54 & M & 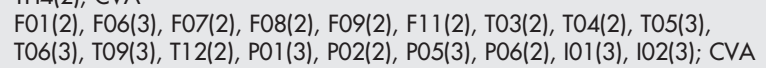 & 3 & 3 \\
\hline RPPL-5 & 60 & M & F06(3), FO7(2), F08(2), FO9(3), F1O(3), PO1(2), PO3(1), I01(3), I02(3); CVA & 3 & 1 \\
\hline RPPL-6 & 35 & $\mathrm{~F}$ & PO4(3); SR & NT & 1 \\
\hline LPPL-1 & 43 & $\mathrm{~F}$ & Left: F02(3), PO4(1), CO2(2), $\mathrm{CO} 3(3)$; Right: $\mathrm{F02}(2), \mathrm{CO} 2(2), \mathrm{CO}(2)$; SR & 1 & 1 \\
\hline LPPL-2 & 48 & M & P01(3), PO2(3), FO6(3), F08(2), 101(3), 102(3); CVA & 1 & 1 \\
\hline LPPL-3 & 66 & M & PO3(1-white); SR & 1 & NT \\
\hline LPPL-4 & 42 & $\mathrm{~F}$ & $\mathrm{F02(2),} \mathrm{F05(2),} \mathrm{P04(2);} \mathrm{CVA}$ & 1 & 1 \\
\hline LPPL-5 & 90 & M & $\mathrm{FO6}(3), \mathrm{FO}(2), \mathrm{FOB}(2), \mathrm{FOO}(3), \mathrm{F1O}(3), \mathrm{PO1}(2), \mathrm{PO}(1), 101(3), 102(3)$; CVA & NT & NT \\
\hline LPPL-6 & 72 & M & $\mathrm{PO1}(2) \mp ; \mathrm{CVA}$ & 1 & 1 \\
\hline LPPL-7 & 76 & M & $\begin{array}{l}\mathrm{PO1}(3), \mathrm{PO} 2(3), \mathrm{FO}(3), \mathrm{FO}(2), \mathrm{TO}(3), \mathrm{TO}(3), \mathrm{TO}(3), \mathrm{TO}(3), \mathrm{TO9}(3), \\
\mathrm{O} 04(2), \mathrm{O} 05(2), \mathrm{I01}(3), \mathrm{IO2}(3) \text {; CVA }\end{array}$ & & \\
\hline LPPL-8 & 52 & $\mathrm{~F}$ & $\mathrm{PO1}(3), \mathrm{PO2}(2), \mathrm{PO} 5(3), \mathrm{TO9}(2) ; \mathrm{SR}$ & 1 & 1 \\
\hline LPPL-9 & 52 & M & $\begin{array}{l}\text { F06(3), FO7(2), F08(2), TO7(2), TO8(3), TO9(3), PO1(1), BG1(3), BG2(3), } \\
\text { BG3(3), IC1(3), IC2(4), IC3(4), IO1(4), IO2(3); CVA }\end{array}$ & NT & NT \\
\hline
\end{tabular}

*Subjects are numbered in each group: RPPL, right posterior parietal lobe lesions; LPPL, left posterior parietal lobe lesions ( $F$, female; $M$, male). †Location of lesion specified by abbreviations as in Damasio and Damasio ${ }^{14}$ : BG, basal ganglia; F, frontal; I, insula; IC, internal capsule; $O$, occipital; $P$, parietal; $\mathrm{T}$, temporal; TH, thalamus.

\pm Size of damaged area in lesion is indicated by the number in parentheses after the location (1, less than $25 \% ; 2,25-75 \% ; 3$, more than $75 \%$ ).

§Cause of lesion: CVA, cerebrovascular accident; SR, surgical resection.

- Neglect rating is from an assessment of the presence and degree of neglect (1, absent; 2, mild; 3, severe), based on neuropsychological testing (Rey-Osterreith complex figure test - copy version, a line cancellation task, a freehand drawing (of a clock)), and the pattern of performance on the Benton visual retention test and judgment of line orientation completed shortly after the lesion (acute) or more than three months after the lesion (chronic).

${ }^{*} \mathrm{NT}$, not tested because no indication of neglect on standard neurological examination. 
Table 2 Ages and lesion locations for individuals with lesions outside the posterior parietal cortex

\begin{tabular}{|c|c|c|c|c|}
\hline Subject & Age (years) & Sex & Side of lesion & Location†, sizeł, and cause $\S$ of lesion \\
\hline LCON-1 & 27 & $M$ & $\mathrm{~L}$ & T05(2); T10(1); T12(3); CVA \\
\hline LCON-2 & 49 & M & $\mathrm{L}$ & T03(1); T08(1); T09(2); CVA \\
\hline LCON-3 & 66 & $\mathrm{~F}$ & L & T04:2; CVA \\
\hline LCON-4 & 65 & $M$ & $L$ & $\mathrm{FO6}(2) ; \mathrm{BG} 1(3)$; $\mathrm{BG} 2(2) ; \mathrm{BG} 3(2)$; IC1(3); IC2(2); IC3(3);CVA \\
\hline LCON-5 & 58 & $M$ & $\mathrm{R}$ & T08(3); T12(2); BG3(2); $101(3) ; 102(1) ;$ CVA \\
\hline LCON-6 & 30 & $\mathrm{~F}$ & $\mathrm{R}$ & T05(1); T10(3); T12(3); SR \\
\hline LCON-7 & 67 & $M$ & $\mathrm{R}$ & T06(1); 001(2); O03(2); CVA \\
\hline LCON-8 & 43 & M & $\mathrm{R}$ & T03(1); T04(2); T08(2); T09(1); CVA \\
\hline \multicolumn{5}{|c|}{$\begin{array}{l}\text { †Location of lesion specified by abbreviations as in Damasio and Damasio }{ }^{14} \text { : BG, basal ganglia; F, frontal; I, } \\
\text { insula; IC, internal capsule; O, occipital; PO, parietal; } T \text {, temporal; } T H \text {, thalamus. } \\
\text { †Size of damaged area in lesion is indicated by the number in parentheses after the location (1, less than } 25 \% ; 2 \text {, } \\
25-75 \% \text {; } 3 \text {, more than } 75 \% \text { ). } \\
\text { §Cause of lesion: CVA, cerebrovascular accident; SR, surgical resection. } \\
\text { F, female; L, left; LCON, lesion control; M, male; R, right. }\end{array}$} \\
\hline
\end{tabular}

typically used in subjects with hemineglect, in which the subject is asked to point-usually with a straight arm-so that the fingertip is placed directly in front of the body midline. ${ }^{14-16}$ Specifically, the task we employed here primarily tests perception of trunk A-P axis direction, whereas the task used in previous studies primarily tests perception of the projection of that axis onto a vertical frontal plane in front of the subject at arm's length distance.

Again, we used two experimental conditions:

(1) standard: five to eight trials with the head in a standard erect posture and arm abducted approximately $90^{\circ}$;

(2) varied: 20 trials with varied head postures (varied head left/right rotation on each trial) and varied shoulder horizontal flexion angles on each trial, such that the elbow angle yielding a forearm position parallel to the trunk A-P axis changed on each trial, so that subjects could not use a remembered elbow angle to complete the task (fig 1).

Initial elbow joint angle was varied on each trial in both conditions. When time permitted, both upper limbs were tested. However, if time constraints prevented testing both limbs in a subject with brain lesion, we tested the limb on the side ipsilateral to the lesion, for reasons given above. Both upper limbs were tested in 13 of 14 controls, as well as in four of seven subjects with lesions outside the posterior parietal lobe, and in eight of 14 subjects with lesions of the posterior parietal lobe.

\section{Data recording}

Orientations of the forearm, arm (humerus), and head were recorded using an electromagnetic system (Ascension Technologies "Flock of Birds"). This system provides 6 degrees of freedom information (three dimensional location and orientation) of a receiver fixed to the body segment of interest relative to an electromagnetic transmitter. The receivers were attached to a plastic adjustable cuff just proximal to the wrist (forearm orientation), over the lateral epicondyle of the humerus in a location where skin movement was minimal, and to an adjustable headpiece that was fitted snugly to the subject's head. The axes of the receivers were aligned to the earth fixed laboratory coordinate system by flexing the upper limb forward to a horizontal position with the elbow extended and the head and trunk in erect positions. The orientations of the body segments were computed relative to this standard orientation.

\section{Data analysis}

Perceptual errors on individual trials were computed from the angular orientation of the forearm relative to the earth fixed vertical (that is, the forearm elevation angle) when aligning the forearm to vertical and relative to the trunk fixed A-P axis (the forearm yaw angle relative to the standard "straight ahead" position defined by the alignment procedure designed above) when moving the forearm in the horizontal plane. We assessed in each subject whether these single trial errors showed a perceptual bias toward the head longitudinal axis (when aligning the forearm to vertical) or the A-P axis (when aligning the forearm to the trunk A-P axis) using single linear regression, as described in previous studies. ${ }^{3}{ }^{4}$

Constant errors were computed as the mean of the single trial errors in each condition (head erect, head orientation varied). Absolute constant errors were computed as the magnitude of the constant error when aligning the forearm to the trunk fixed A-P axis. Variable errors were computed as the standard deviation of the single trial errors in each condition. In the Results section, we present mean errors for the different groups to allow general comparisons among groups, but did not use analysis of variance techniques because the brain lesions of different subjects within each of the LCON (lesion control), LPPL (left posterior parietal lobe lesion), and RPPL (right posterior parietal lobe lesion) groups differed in size and location (tables 1 and 2). Instead, we used stepwise multiple regression techniques to evaluate whether lesions of different size and location differentially affected perceptual errors.

We used simple linear correlation to test whether errors increased with age in our subjects. This is because previous work has shown declines in vestibular and proprioceptive senses with age. ${ }^{17-19}$

The dependent variables in these multiple regression analyses were the constant and variable errors for a particular condition, with independent variables being the location and size of the brain lesions in different regions of the posterior parietal lobe (supramarginal gyrus, angular gyrus, and superior parietal lobule) as well as in other brain regions. Inferior parietal lobule lesion volumes were the sum of P01 (supramarginal gyrus) and P02 (angular gyrus) lesion volumes. SPL lesion volumes were quantified as the sum of P03 (lateral areas 5, 7) plus P04 (mesial areas 5, 7) plus P05 (paraventricular area) plus P06 (supraventricular area) lesion volumes. The posterior insula (I02) lesion volume was also considered because previous studies have implicated this region in visual perception of vertical. ${ }^{20}{ }^{21}$ Volumes of posterior superior temporal gyrus lesions (PSTG, cell T09) were also considered because a recent study has implicated this region in hemineglect. ${ }^{11}$ Volume of lesions in cells F06 and F08 were considered because functional MRI research has shown that this region is involved in visual perception of the mid-sagittal plane or "straight ahead" direction. Total lesion volume outside PPL, F06-F08, PSTG, and posterior insula (that is, the sum of all lesion volumes outside these regions) was also included as an independent variable to test whether lesions outside these regions influenced errors. Lesion volumes were 
quantified within each cell of the inferior and posterior parietal lobules, I02, F06-F08, and other brain areas, as 0 (no lesion), 1 (up to $25 \%$ of region), 2 (25-75\% of region), or 3 (75-100\% of region). ${ }^{22}$ Thus, I02, P01, and P02 lesion volumes could range from 0-3, F06-F08 lesion volumes from 0-6, and SPL lesion volumes from $0-12$.

The multiple regression analysis initially included volumes of lesions to PPL (sum of SPL and IPL lesion volumes), posterior insula (I02), frontal motor/premotor areas (F06F08), PSTG (T09), and total volume of lesions outside these areas as independent variables. If this initial regression produced no significant results, we undertook a stepwise regression analysis using lesion volumes of only IPL and SPL. If PPL or SPL and IPL lesion volumes contributed significantly to prediction of the errors $(p<0.05)$, then a second regression was run with lesion volumes of supramarginal and angular gyri (P01, P02) or SPL and other significant independent variables from the initial regression. Although side of the lesion may affect perceptual errors, it was not used as an independent variable in the multiple regression analysis because it is a categorical and not a continuous variable. Multiple regression analysis was also used to determine whether the errors on individual trials (dependent variable) depended on head and shoulder orientation.

\section{RESULTS}

\section{Perception of vertical}

Lesions of the posterior parietal lobe had little influence on constant errors for aligning the forearm to vertical in 13 of the 15 subjects with posterior parietal lobe lesions (fig 2A). Constant errors averaged about 0.3 rad and were similar among controls and subjects with lesions (fig 2A). These constant errors reflect deviation from vertical of the sensor mounted on the forearm, which is affected by alignment of the sensor with the longitudinal axis of the forearm. Age did not influence constant errors $(p=0.59)$. Constant errors were usually lower when head and shoulder orientations were varied, except for two subjects with right PPL lesions (RPPL4, RPPL-6) who had very large constant errors (0.4-0.9 rad) when the head was erect, but not when head orientation was varied.

Most subjects with right PPL lesions made larger variable errors than normal controls and lesion controls with the left upper limb only (fig 2B). Age influenced variable errors $(p=0.036)$, but correlation coefficients within groups were quite variable and, surprisingly, often negative, indicating lower errors in older individuals. For example, among controls, variable errors were negatively correlated with age for the right arm under standard conditions $(r=-0.64)$ and for the left arm under head and shoulder varied orientations $(r=-0.8)$, but were poorly correlated with age for other conditions $(\mathrm{p}>0.05)$. Age did not affect variable errors in a consistent manner for any of the groups. As expected, varying head and shoulder orientations increased variable errors similarly in all groups and each arm (fig 2B).

Perceptual errors on individual trials depended on orientations of the arm and head in all groups of subjects. Mean multiple correlation coefficients ranged from 0.5 to 0.6 and were statistically similar for the two arms and the four groups of subjects $(p>0.29)$. However, there was high intersubject variability in all groups as the correlation coefficients ranged from 0.035 to 0.91 , indicating that some subjects' errors depended strongly on head or arm orientation while others did not. Also there was no indication that subjects with posterior parietal lobe lesions tended to align the forearm toward or away from the head longitudinal axis, as most of these subjects did not have a significant correlation between forearm positioning errors and frontal plane head orientation. Instead, errors depended primarily on small variations in head flexion/extension and rotation.

Multiple regression analysis showed that higher variable and constant errors with both arms were usually associated with greater lesion volumes in the right and left superior parietal lobules. Constant errors increased with SPL lesions when the task was done with the head erect $(r=0.578$, $\mathrm{p}=0.003$ for left arm; $r=0.335, \mathrm{p}=0.075$ for right arm; $p>0.25$ for lesion volumes of other brain areas), but not when head and shoulder orientations were varied $(p>0.13)$. Variable errors increased with larger lesions of the right and left SPL when aligning the left forearm to vertical with the head erect $(r=0.568, \mathrm{p}=0.001)$ and when head orientation was varied (fig 3; $r=0.66, \mathrm{p}<0.001$ ). Larger variable errors were also observed in subjects with larger lesions of the left and right SPL when aligning the right forearm to vertical when head orientation was varied (fig $3 ; r=0.608, \mathrm{p}<0.001$ ), but when the head was erect, variable errors depended on the volume of angular gyrus lesions $(r=0.408, \mathrm{p}=0.025)$. Variable errors showed no significant dependence on lesions of other areas of the brain.

\section{Perception of the trunk A-P axis}

Most subjects with lesions of the posterior parietal lobule aligned the forearm to the trunk fixed A-P axis with constant errors similar to those of controls. Constant and absolute constant errors did not differ among the subject groups (fig 4A), although some subjects with PPL lesions had large constant errors, as indicated by the high intersubject variability in these groups (fig 4A). Notably, subject LPPL5-who had a lesion of the left PPL and a small lesion to the left sensorimotor cortex and premotor areas (table 1) - had a very large constant error of $0.75 \mathrm{rad}$ when using the right arm with the head erect and aligned to the trunk. The constant errors showed a significant dependence on age $(p=0.022)$, but a range of significant positive and negative correlations was observed in the different groups owing to positive and negative constant errors (fig 4A). In contrast, absolute constant errors showed no significant dependence on age $(\mathrm{p}=0.24)$.

Most subjects with posterior parietal lobe lesions had larger variable errors than controls, especially when head and shoulder orientations were varied. Variable errors were generally larger in the left and right PPL group than in normal controls (fig 4C). Varying head and shoulder orientations increased variable errors in all groups, as expected. The right and left PPL groups generally had larger increases in variable errors than the normal controls owing to varied head and shoulder orientations. The variable errors showed no significant dependence on age $(p=0.82)$.

Errors on individual trials depended on head and shoulder orientations in all subject groups. Multiple correlation coefficients for dependence of errors on shoulder orientations were greater than 0.5 in most subjects and exceeded 0.9 in some. Dependence of errors on head orientations was somewhat less, but the multiple correlation coefficients exceeded 0.4 in most subjects and exceeded 0.8 in some. Average multiple correlation coefficients were similar in all groups and for both arms $(\mathrm{p}>0.104)$. Thus both controls and subjects with brain lesions have similarly strong relations between perceptual errors and varied head and trunk orientations. There was no strong evidence for a perceptual bias toward the head fixed A-P axis when aligning the forearm to the trunk fixed A-P axis in controls or in subjects with PPL damage, because correlation coefficients for the relation between single trial perceptual errors and head rotation angles were not significant in most subjects.

The magnitudes of constant and variable errors depended on lesion volumes of various brain areas. Absolute constant 

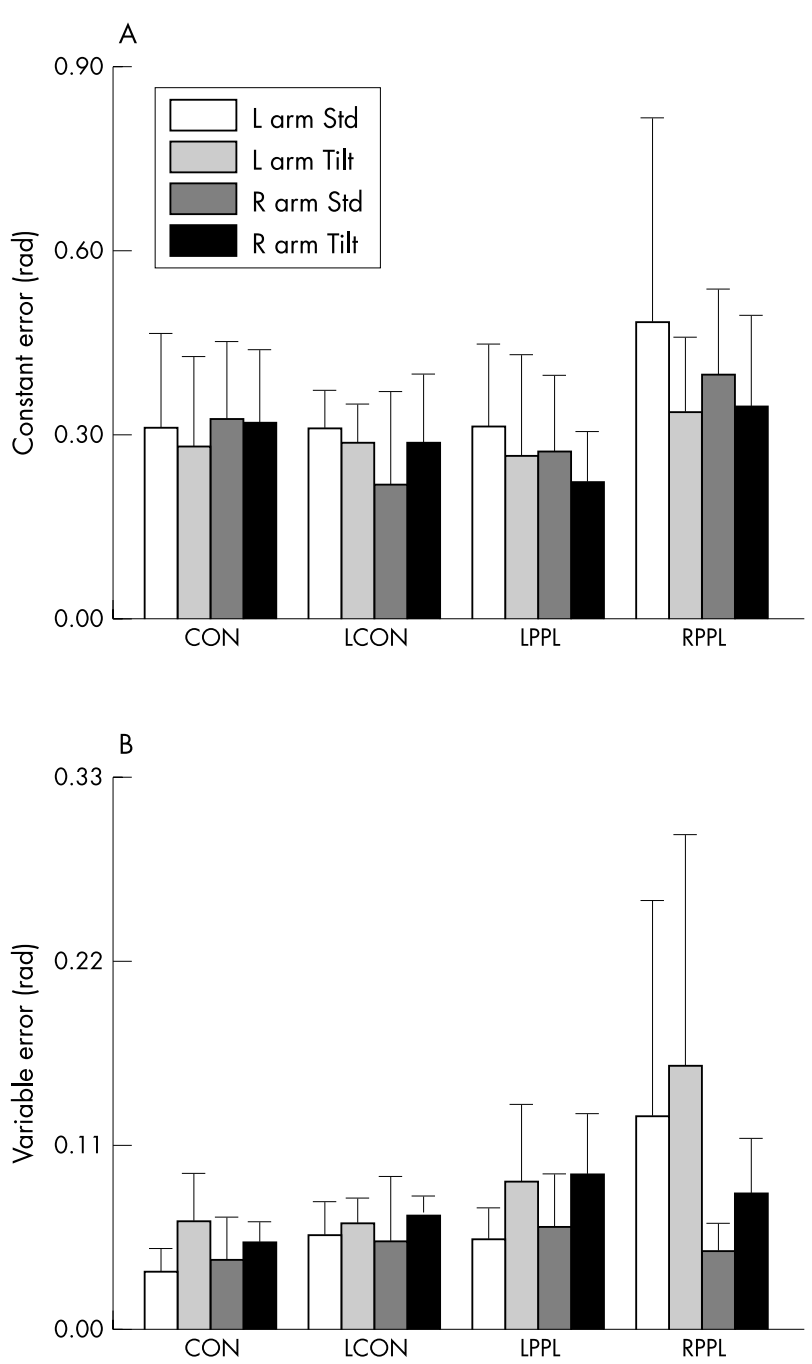

Figure 2 Mean errors for aligning the forearm to the earth fixed vertical. Average constant (A) and variable (B) elevation angle errors are shown for each group of subjects. Abbreviations on the abscissae represent the group (CON, controls; LCON, lesion controls; LPPL, left posterior parietal lobe lesions; RPPL, right posterior parietal lobe lesions). Different types of bar show errors for aligning the left versus right forearm to vertical and whether the head was in the standard erect position (Std) or varied in orientation by left/right lateral flexion (Tilt). Error bars are 1 SD.

errors for the left upper limb showed no significant dependence on lesion volumes in any brain areas, although larger PSTG lesions were usually associated with larger errors when head and shoulder orientations were varied $(\mathrm{p}=0.08)$. In contrast, absolute constant errors for the right arm were strongly related to volume of lesions in premotor areas, inferior parietal lobule, and PSTG. When head and trunk were normally aligned, absolute constant errors increased with larger premotor (FO6-FO8) lesions $(\mathrm{p}<0.001)$, but paradoxically decreased with larger PSTG lesions $(\mathrm{p}<0.001)$ in this condition only. When head and shoulder orientations were varied, right arm absolute constant errors increased with volume of angular and supramarginal gyrus lesions $(r=0.5, \quad \mathrm{p}=0.02 ; \mathrm{p}=0.006$ for supramarginal gyrus, $\mathrm{p}=0.069$ for angular gyrus), but were not related to size of PSTG lesions $(\mathrm{p}>0.1)$.

Variable errors also usually increased with volume of SPL lesions. When head and trunk were normally aligned, left upper limb variable errors increased with volume of lesions to SPL $(p=0.063)$ and premotor areas $(p=0.033)$, with a

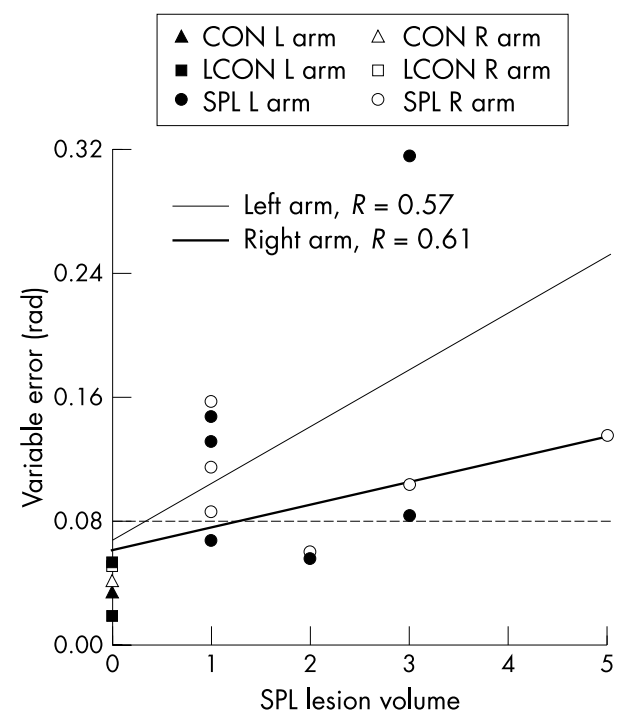

Figure 3 Dependence of variable errors for perception of forearm alignment to the earth fixed vertical on lesions of superior parietal lobule (SPL). The scatterplot shows variable errors for the right arm (empty symbols) and the left arm (filled symbols) with the head maintained in the upright position for individual subjects with focal lesions of the SPL, and mean errors for controls and lesion controls (CON, LCON, empty symbols). The oblique solid lines represent the relations between SPL lesion volume and variable errors for left and right arms from the regression equations using data from all subjects. The horizontal dashed line represents 2 SD above the mean of normal controls. Variable errors higher than the dashed line would be classified as abnormally large.

multiple correlation coefficient of $0.466(p=0.029)$, but errors with the right upper limb depended on volume of lesions outside the PPL $(r=0.58, \mathrm{p}<0.01)$. When head and shoulder orientations were varied, variable errors increased with volume of SPL lesions $(\mathrm{p}<0.003)$ and, for the right arm, they also increased with larger supramarginal gyrus lesions $(\mathrm{p}=0.043)$, with multiple correlation coefficients of 0.66 and 0.71 for left and right arms, respectively (fig 5). They were not related to size of PSTG lesions $(p>0.1)$.

\section{DISCUSSION}

Consistent with our hypothesis, we found that larger unilateral lesions of the superior parietal lobules produce larger variable errors in aligning the unseen forearm to the earth fixed vertical and the trunk fixed A-P axis. Thus both the right and left SPLs contribute to perception of right and left forearm orientation relative to these axes. Lesions of other brain areas generally had little effect on errors for aligning the forearm to vertical, although variable errors for aligning the right arm to vertical tended to increase with lesion volume of the right and left angular gyri of the inferior parietal lobule. Furthermore, larger unilateral lesions of premotor areas and the inferior parietal lobules were associated with larger magnitude constant errors for aligning the forearm to the trunk A-P axis. This finding is consistent with the idea that a network involving these brain areas is critical for accurate perception of upper limb orientation in the horizontal plane. Imposed variations of head and shoulder orientation increased errors in aligning the forearm to both axes in all subjects. The increased errors on individual trials depended on the variations of head and shoulder orientations.

The finding that lesions of the right and left SPLs induced deficits in aligning the right and left forearms to the earth fixed vertical is consistent with the extensive sensory inputs from the primary somatosensory area and motor and visual 

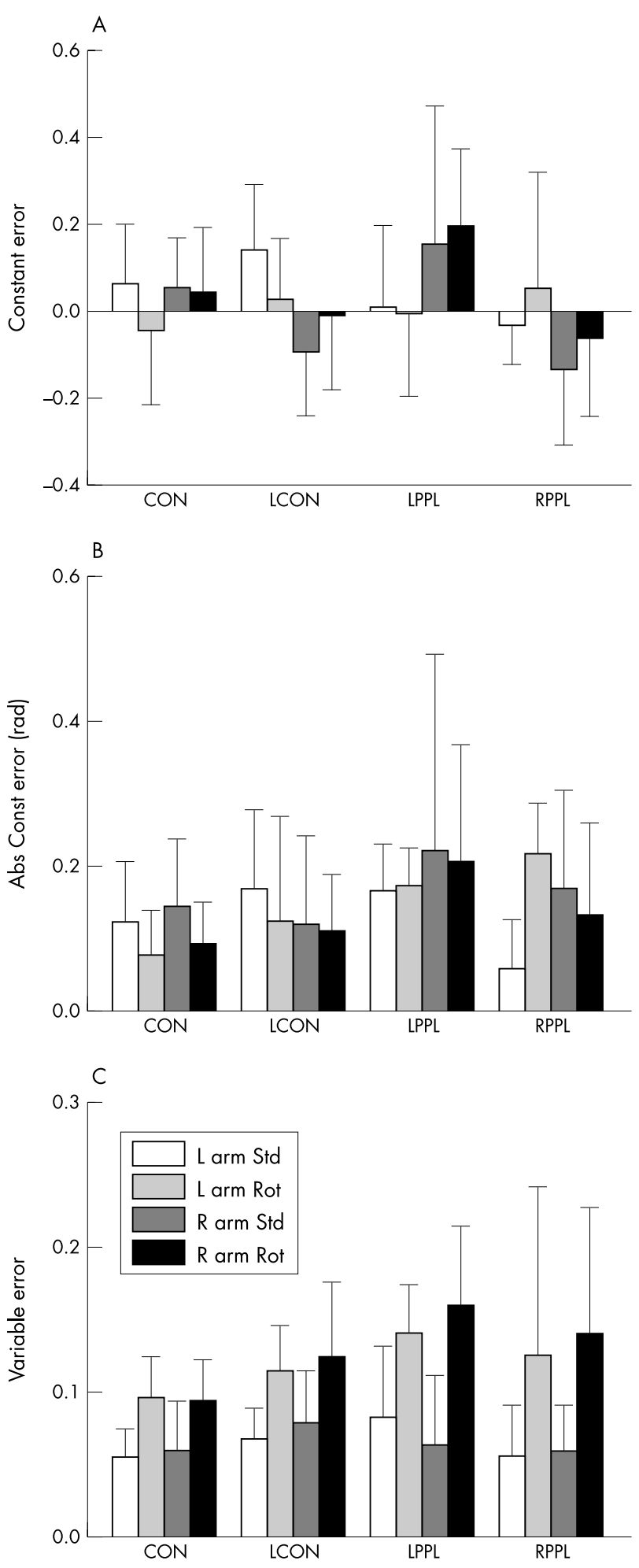

Figure 4 Mean errors for kinaesthetic perception of the trunk fixed anterior-posterior (A-P) axis. Average constant (A), absolute constant (B), and average variable errors (C) are plotted for each group of subjects. Abbreviations on the abscissae and in the legend represent the group (CON, controls; LCON, lesion controls; LPPL, left posterior parietal lobule lesions; RPPL, right posterior parietal lobule lesions) and the experimental conditions (Std, standard head erect position; Rot, head rotated to left/right). Different types of bar show errors for aligning the left and right forearm to the trunk A-P axis under standard (Std) and varied (Rot) head orientation conditions.

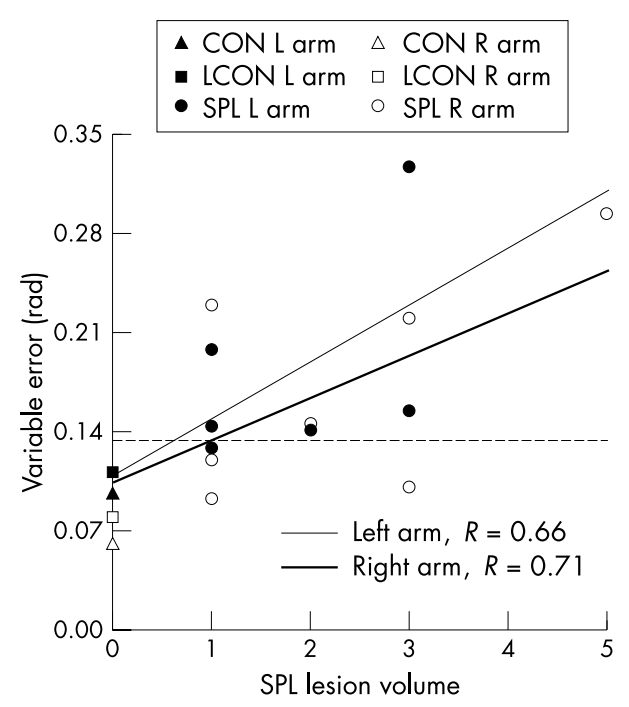

Figure 5 Dependence of variable errors for perception of the trunk fixed anterior-posterior (A-P) axis on lesions of the superior parietal lobule (SPL). The scatterplot shows variable errors for right and left arms (filled symbols) with the head orientation varied for individual subjects with focal lesions of the SPL (left (L) and right (R) arms) and mean errors for controls and lesion controls (CON, LCON, empty symbols). The oblique solid lines represent the relations between SPL lesion volume and variable errors for left and right arms from the regression equations, using data from all subjects. The horizontal dashed line represents 2 SD above the mean of normal controls. Variable errors higher than the dashed line would be classified as abnormally large.

areas to this region of the brain. ${ }^{23}$ A specific role for the left SPL in visual and kinaesthetic perception related to the vertical was indicated by previous findings that the left, but not the right, SPL was activated by galvanic vestibular stimulation, which stimulates the vestibular organs. ${ }^{24}$ The left secondary somatosensory area also appears to be more important in processing of proprioceptive sensory input than the right, ${ }^{25}$ suggesting that the left parietal lobe may dominate in proprioceptive processing. However, two of our subjects with large right SPL lesions showed high variable errors when aligning the left or right forearm to vertical. Thus lesions to the right SPL can also influence kinaesthetic perception of the vertical for both upper limbs. Because there were only seven subjects with PPL lesions that included the SPL, these conclusions would be strengthened by similar findings in a larger group of subjects with SPL lesions.

There was no evidence that the posterior insula played a role in accurate aligning of the forearm to the earth fixed vertical. Multiple regression analyses did not show a significant dependence of constant or variable errors on size of posterior insula lesions. Also, an examination of individual subject data showed that moderate to large lesions of this region did not adversely affect kinaesthetic perception of the vertical. For example, subjects LPPL-7 and RPPL-1 had PPL and I02 lesions but had similar magnitudes of constant and variable errors (range 0.02 to $0.13 \mathrm{rad}$ ) to those of LPPL- 6 and RPPL-3 (range 0.02 to $0.1 \mathrm{rad}$ ), who had no posterior insula lesions. Thus kinaesthetic perception of the vertical either does not depend on processing of vestibular information by the insula, or other compensatory mechanisms are used in the absence of such processing. Possible compensatory mechanisms include the use of trunk graviceptors (statoliths, blood distribution ${ }^{26}{ }^{27}$ ) or sensory information concerning gravitational torques about the elbow ${ }^{28}$ to specify vertical. Alternatively, cerebral cortex reorganisation following lesions may allow processing of vestibular and other sensory inputs by non-insular cortical regions to permit accurate 
kinaesthetic perception of the vertical. Thus, although the posterior insula apparently processes inputs from the vestibular otoliths ${ }^{20} 24$ and acute lesions of parieto-insularvestibular cortex affect visual perception of the vertical, ${ }^{21}$ our findings suggest that this area does not play a critical role in kinaesthetic perception of the earth fixed vertical axis. This conclusion is also consistent with findings that parietoinsular vestibular cortex (PIVC) neurones respond strongly to head/ body rotation but not to static tilt. ${ }^{29}$ We recently showed that chronic stage lesions involving the posterior insula do not cause larger errors in visual perception of the vertical, ${ }^{30}$ also suggesting that this region is not critical for defining the earth fixed vertical axis at the perceptual level.

There were no consistent effects of lesions involving inferior parietal lobule and posterior regions of the superior temporal gyrus on errors for kinaesthetic perception of the trunk fixed A-P axis direction. Larger lesions of these areas were associated with increased perceptual constant and variable errors in limited conditions only. Similarly, larger lesions of FO6-FO8 cells (including areas 6 and 44) produced larger constant errors only when aligning the right forearm to the trunk A-P axis when head and trunk were aligned. There were no such effects in other conditions. The finding that larger lesions of the PSTG were not associated with larger errors in aligning the forearm to the trunk A-P axis raises the question of whether PSTG lesions affect these abilities in the absence of hemineglect.

The SPLs in both hemispheres contribute to kinaesthetic perception of the trunk fixed A-P axis, based on the finding of consistently larger variable errors for aligning both forearms to the trunk A-P axis with larger lesions of the right and left SPLs. Larger lesions of F06 and F08 cells and supramarginal gyrus (P01) were also associated with larger variable errors, but in only one condition each. Previous work indicated that accurate visual perception of "straight ahead" depends on a bihemispheric fronto-parietal network involving areas 6 and 44. ${ }^{12}$ However, we recently observed that unilateral lesions in those areas did not disrupt direct visual perception of the trunk A-P axis direction. ${ }^{30}$ Thus kinaesthetic perception is disrupted by such unilateral lesions, along with visual processing for voluntary eye movement control, but visual perception of the straight ahead direction appears to be relatively unaffected if the lesion is unilateral.

Unilateral SPL lesions may disrupt processing of shoulder proprioceptive inputs needed for accurate alignment of the forearm to upper limb kinaesthetic coordinate system axes. Multiple correlation coefficients for relations between kinaesthetic perceptual errors on individual trials and shoulder orientations were similar in controls and subjects with SPL lesions, but variable errors for forearm alignments to the axes were larger in most subjects with SPL lesions, indicating larger single trial errors. Accurate positioning of the forearm in these tasks probably requires the integration of proprioceptive inputs from muscle, skin, and joint afferents that indicate shoulder and elbow joint angles. Thus these results suggest an inability to compensate adequately for different shoulder orientations when varied by the experimenter. However, inability to compensate for the effects of varied head orientations on perception of vertical and trunk A-P axes may also play a role. Study of separate effects of varying shoulder versus head orientations is needed to assess whether both play a role or whether the higher variable errors primarily reflect variations in head or shoulder orientations.

Our findings are consistent with those of a recent study in a large group of individuals $(n=23)$ with left hemineglect, which did not find evidence of ipsilesional deviations in proprioceptively guided pointing "straight ahead," when the initial position of the pointing hand was aligned to the midline. ${ }^{31}$ The hand was initially positioned in right (nonneglected) hemispace in previous work demonstrating ipsilesional deviations. ${ }^{14}{ }^{15}$ However, the methods of this previous research involved pointing to place the fingertip directly in front of body midline, rather than aligning the forearm parallel to the trunk A-P axis as in the present work. Errors on the proprioceptive pointing task used previously ${ }^{16}{ }^{17}$ have been interpreted to reflect displacement of the trunk midline reference to the right or left, but may also reflect rotation of the trunk fixed A-P axis or "straight ahead" reference. Errors in the forearm positioning task used in this study are probably related primarily to rotation of the trunk fixed A-P axis reference, because our subjects were instructed to position the forearm parallel to the reference axis.

\section{Conclusions}

We found that individuals with lesions involving the superior parietal lobule showed large constant or variable errors in perception of vertical and trunk fixed A-P axis direction. This is consistent with the idea that the SPL functions as an area that defines higher level coordinate systems for the kinaesthetic system and for use in programming reaching movements to visual targets. The angular gyrus and frontal premotor areas (F06-F08 cells) may also be involved in higher level kinaesthetic perception, as indicated by the multiple regression analysis, but their effects were less consistent than those of SPL lesions. Study of a larger group of subjects with lesions of these brain areas is needed to assess whether they make significant contributions to kinaesthetic perception of forearm orientation in earth fixed and trunk fixed frames of reference.

\section{ACKNOWLEDGEMENTS}

This research was supported by NIH grants PO NS19632 and NS34895.

\section{Authors' affiliations}

W G Darling, R Bartelt, Department of Exercise Science, Department of Physical Medicine and Rehabilitation, Program in Neuroscience, University of lowa, lowa City, lowa, USA

M Rizzo, Division of Behavioral Neurology and Cognitive Neuroscience, Department of Neurology, Program in Neuroscience, University of lowa

Competing interests: none declared

\section{REFERENCES}

1 Gordon J, Ghilardi MF, Ghez C. Impairments of reaching movements in patients without proprioception. 1. Spatial errors. J Neurophysiol 1995:73:347-60.

2 Ghez C, Gordon J, Ghilardi MF. Impairments of reaching movements in patients without proprioception .2. Effects of visual information on accuracy. J Neurophysiol 1995;73:361-72.

3 Darling WG, Williams TE. Kinesthetic perceptions of intrinsic anteriorposterior axes. Exp Brain Res 1997;1 17:465-71.

4 Darling WG, Hondzinski JM. Kinesthetic perceptions of earth- and body-fixed axes. Exp Brain Res 1999;126:417-30.

5 Darling WG, Hondzinski JM. Visual perceptions of vertical and intrinsic longitudinal axes. Exp Brain Res 1997;116:485-92.

6 Darling WG, Butler AJ, Williams TE. Visual perceptions of head-fixed and trunk-fixed anterior/posterior axes. Exp Brain Res $1996 ; 112: 127-34$

7 Soechting JF, Flanders $M$. Sensorimotor representations for pointing to targets in 3-D space. J Neurophysiol 1989;62:582-94.

8 Soechting JF, Flanders $M$. Errors in pointing are due to approximations in transformations. J Neurophysiol 1989;62:595-608.

9 Flanders M, Tillery SIH, Soechting JF. Early stages in a sensorimotor transformation. Behav Brain Sci 1992;15:309-61.

10 Binkofski $F$, Buccino $G$, Posse $S$, et al. A fronto-parietal circuit for object manipulation in man: evidence from an fMRI-study. Eur J Neurosci 1999;11:3276-86.

11 Karnath HO, Ferber S, Himmelbach M. Spatial awareness is a function of the temporal not the posterior parietal lobe. Nature $2001 ; 411: 950-3$. 
12 Vallar G, Lobel E, Galati G, et al. A fronto-parietal system for computing the egocentric spatial frame of reference in humans. Exp Brain Res 1999; 124:281-6.

13 Galati G, Lobel E, Vallar G, et al. The neural basis of egocentric and allocentric coding of space in humans: a functional magnetic resonance study. Exp Brain Res 2000;133:156-64.

14 Heilman KM, Bowers D, Watson RT. Performance on hemispatial pointing task by patients with neglect syndrome. Neurology 1983;33:661-4.

15 Chokron S, Bartolomeo P. Position of the egocentric reference and directional arm movements in right-brain-damaged patients. Brain Cogn 1998;37:405-18.

16 Karnath $\mathrm{H}-\mathrm{O}$. Subjective body orientation in neglect and the interactive contribution of neck muscle proprioception and vestibular stimulation. Brain 1994:117:1001-12.

17 Schweigart G, Chien RD, Mergner T. Neck proprioception compensates for age-related deterioration of vestibular self-motion perception. Exp Brain Res 2002; 147:89-97.

18 Pai YC, Rymer WZ, Chang RW, et al. Effect of age and osteoarthritis on knee proprioception. Arthritis Rheum 1997;40:2260-5.

19 Bullock-Saxton JE, Wong WJ, Hogan N. The influence of age on weightbearing joint reposition sense of the knee. Exp Brain Res 2001;136:400-6.

20 Bucher SF, Dieterich $M$, Wiesmann $M$, et al. Cerebral functional magnetic resonance imaging of vestibular, auditory, and nociceptive areas during galvanic stimulation. Ann Neurol 1998;44:120-5.
21 Brandt $T$, Dieterich $M$, Danek A. Vestibular cortex lesions affect the perception of verticality. Ann Neurol 1994;35:403-12.

22 Damasio H, Frank R. Three-dimensional in vivo mapping of brain lesions in humans. Arch Neurol 1992;49:137-43.

23 Stein JF. The representation of egocentric space in the posterior parietal cortex. Behav Brain Sci 1992;15:691-700.

24 Lobel E, et al. Functional MRI of galvanic vestibular stimulation. J Neurophysiol 1998;80:2699-709.

25 Simoes CM, Alary F, Forss N, et al. Left-hemisphere dominance of the human SIl activation after bilateral median nerve stimulation. Soc Neurosci Abstr $2001 ; 27$.

26 Mittelstaedt H. Somatic graviception. Biol Psychol 1996;42:53-74.

27 Mittelstaedt $\mathbf{H}$. Evidence of somatic graviception from new and classical investigations. Acta Otolaryngol Suppl 1995;520:186-7.

28 Worringham CJ, Stelmach GE. The contribution of gravitational torques to limb position sense. Exp Brain Res 1985;61:38-42.

29 Akbarian S, Berndl K, Grusser OJ, et al. Responses of single neurons in the parietoinsular vestibular cortex of primates. Ann NY Acad Sci 1988; 545:187-202.

30 Darling WG, Pizzimenti MA, Rizzo M. Unilateral posterior parietal lobe lesions affect representation of visual space. Vis Res 2003;43:1675-88

31 Farne A, Ponti F, Ladavas E. In search of biased egocentric reference frames in neglect. Neuropsychologia 1998;36:611-23. 